\title{
Generalization of visual matching and delayed matching by a California sea lion (Zalophus californianus)
}

\author{
ADAM A. PACK, LOUIS M. HERMAN, and HERBERT L. ROITBLAT \\ University of Hawaii, Honolulu, Hawaii
}

\begin{abstract}
Only a limited number of species have been found capable of generalized matching-to-sample (MTS) after exposure to relatively few training exemplars. We trained a juvenile, experimentally naive California sea lion (Zalophus californianus) in MTS, using a pair of three-dimensional objects as samples. Successful matching to a criterion of $90 \%$ correct or better over 2 successive sessions was attained in 12 sessions (269 trials and 70 errors). Two subsequent "partial" transfer tests, in which each of the two training objects was paired with a novel test object, and four additional transfer tests, all with novel objects, were presented following training. An $80 \%$ performance criterion over 2 successive sessions was reached, or closely approximated, in from 2 to 4 transfer sessions for all transfer tests; errors to criterion tended to be reduced across the successive novel transfer tests and were as few as five during the final two tests; and performance on the first 48 trials of the last two novel transfers was not significantly different from a nearceiling level baseline performance measure. Neophobic responses of the sea lion to new objects precluded an unbiased evaluation of immediate (Trial 1) transfer. The sea lion's short-term memory for sample objects was also measured. Matching performance was maintained at a level of $78 \%$ correct responses or better for delays through to $45 \mathrm{sec}$ after removal of the sample object. At a 58-sec delay, the longest tested, performance declined to $69 \%$ correct responses. These retention levels are only somewhat below levels reported for dolphins and nonhuman primates tested on visual delayed MTS, but they are above levels typically reported for pigeon subjects.
\end{abstract}

A fundamental issue in comparative cognition is the ability of animals to form broad, general concepts about the relationships among objects or other types of stimuli. Although this ability may be tested within many different paradigms (see Roitblat, 1987, for a review), tasks that direct the animal to detect and report on the identity relationship have been particularly useful. These include same/different tasks, in which the animal judges whether or not two items are identical (e.g., Wright, 1971), as well as matching-to-sample (MTS) tasks (e.g., D'Amato, 1973). There are several forms of the MTS task (cf. D'Amato \& Worsham, 1974; Herman \& Thompson, 1982; Roitblat \& Scopatz, 1983); in this paper, we will

This research was supported in part by Contract N00014-85-K-0210 from the Office of Naval Research to L. M. Herman and by a grant from Earthwatch. We are grateful to Sea Life Park, Oahu, and their former director of training, Ingrid Shallenberger, for generosity in providing research space, the research animal, and much help in the care of the sea lion. Kathy Sdao helped greatly throughout the testing of the sea lion, and Anne Windsor provided valuable assistance during Experiment 2. An earlier version of this paper was presented at the 1987 meeting of the Animal Behavior Society in Williamstown, MA. A. A. Pack and L. M. Herman are affiliated with both the Kewalo Basin Marine Mammal Laboratory and the Department of Psychology, and H. L. Roitblat with the Department of Psychology, at the University of Hawaii in Honolulu. Requests for reprints should be addressed to Louis M. Herman, Kewalo Basin Marine Mammal Laboratory, University of Hawaii, 1129 Ala Moana Blvd., Honolulu, HI 96814. report on visual identity MTS. This task is typified by three steps: (1) the exposure, usually brief, of a sample stimulus, followed by the stimulus' removal; (2) the interposition of a delay period, ranging from $0 \mathrm{sec}$ upwards; and (3) the exposure of two or more alternatives, one of which physically matches the sample. The animal is rewarded for choosing the matching alternative.

To measure the development of a concept of identity, the experimenter presents new stimulus items after MTS training has been completed with a limited number of items; the experimenter then tests whether or not the subject can transfer the identity rule by applying it to these new items. Transfer is evidenced most convincingly by the immediate solution of new MTS problems-that is, by successful matching of new materials on the first trial or the first few unique trials of a new problem. Rapid but not immediate solution provides weaker yet still useful evidence, especially if it can be shown that extraneous factors, such as perceptual limitations or emotional responses (e.g., neophobic responses to new stimulisee D'Amato, Salmon, \& Colombo, 1985; Jackson \& Pegram, 1970; Zentall \& Hogan, 1978), may have impeded immediate transfer. Additionally, it must be shown that the transfer was not simply the result of general improvement through MTS practice or of other broad, general learning factors such as enhanced attention to relevant stimulus dimensions (see discussion in D'Amato et al., 1985). 
Premack (1983) has claimed that the identity concept is widespread among species. Data indicate, however, that the relative abstractness of the concept-the degree to which it can be applied readily to new exemplars or new dimensions-can vary widely among species. Chimpanzees (Nissen, Blum, \& Blum, 1948; Oden, Thompson, \& Premack, 1988) and bottlenosed dolphins (Herman \& Gordon, 1974; Herman, Hovancik, Gory, \& Bradshaw, 1989; Hunter, 1988) are capable of immediate transfer of the identity concept, as evidenced by their consistently high levels of performance on the first trial of new matching problems. In these species, the identity concept is robust; for example, spontaneous transfer of a matching rule to new objects has been demonstrated in infant chimpanzees (Oden et al., 1988), and transfer of a matching rule has been demonstrated in dolphins with both auditory and visual materials (Herman \& Gordon, 1974; Herman et al., 1989).

Old- and new-world monkeys evidence a somewhat reduced capability for rapid transfer of a matching rule, in comparison with chimpanzees or dolphins. Thus, when Mello (1971) gave extensive MTS training (ca. 150 days, or 7,500 trials) with form stimuli to 3 rhesus monkeys (Macaca mulatta), there was no evidence of immediate transfer to four pairs of novel forms, although overall performance during each day of testing generally remained only slightly below the baseline level established during training. Also, in Weinstein's (1941) study, first-trial transfer to novel objects was not obtained with 2 rhesus monkeys after training with only a single pair of objects, but it was obtained after additional training with 24 new objects paired in various combinations. D'Amato and Colombo (1989) have reported that cebus monkeys ( $\mathrm{Ce}$ bus apella) are limited in the degree to which the identity concept is transferable to new classes of stimuli. Monkeys with extensive previous experience in matching twodimensional visual stimuli, or in making sameldifferent judgments with these stimuli, showed no transfer of the matching or same/different rules to flashing versus steadystate colored disks-that is, they were not able to transfer the identity concept to a new stimulus dimension. An additional problem in assessing the facility or rapidity in transferring the matching concept to new materials is the fear response to novel stimuli seen in some monkeys (see, e.g., D'Amato et al., 1985).

Some researchers have tested for what may be termed partial transfers, after training with a limited set of items (usually less than four). Partial transfers consist of new pairings of familiar items, or the pairing of a novel item with a familiar item. Using the latter procedure, D'Amato (1971) demonstrated first-session transfer in cebus monkeys, using form stimuli, ${ }^{1}$ and Jackson and Pegram (1970) demonstrated first-trial transfer in rhesus monkeys, using color stimuli. In general, partial transfer seems a considerably simpler task than a contrast between two novel items (cf. Mello, 1971). It is nevertheless a useful measure, since some animals may be capable of partial transfers but not of wholly novel transfers. In summary, research indicates that monkeys can acquire a matching concept, but that it appears to be more limited in its application than is the case with apes or dolphins. Monkeys may approach the immediate-transfer performance of apes and dolphins only after exposure to a relatively large set of exemplars and, possibly, only after neophobic responses have abated.

Reliable transfer of the matching rule has been difficult to demonstrate in pigeons (see reviews in D'Amato et al., 1985; Premack, 1978). In some cases (e.g., Zentall \& Hogan, 1978), neophobic responses have also been a problem in the study of pigeons. Significant first-trial transfer appears to have been reported in only one study (Wright, Cook, River, Sands, \& Delius, 1988). Extraordinary procedures were required, however: over 27,000 training trials in which a pool of 232 unique full-color cartoon figures served as sample and distractor stimuli. Each trial of each 76-trial training session was conducted with a different pair of stimuli. Only during the second transfer test, which consisted of 40 novel pairs not seen previously, did performance reach the approximate $80 \%$ level of the baseline condition (consisting of repetitions of the original training stimuli in random pairings). Wright et al. (1988) suggested that pigeons may be more prone to encode stimuli-specific associations than to form a generalized rule about same/different relationships; the method used to train matching is thus critical to the attempt to determine whether or not a generalized concept is acquired. The method may be more critical for pigeons than for nonhuman primates or dolphins, however, since generalized matching in the latter species has been obtained with sample sets of limited size, with many different procedures, with short sample durations, and with different stimulus modalities (e.g., Colombo \& D'Amato, 1986; D'Amato \& Worsham, 1972; Herman \& Gordon, 1974; Herman et al., 1989; Herman \& Thompson, 1982; Nissen et al., 1948; Oden et al., 1988). Also, performance levels on transfer tests at or near the ceiling level of $100 \%$ correct responses have been observed in these mammalian species in several of the same studies.

Other than dolphins, the only marine mammals tested for MTS have been pinnipeds (seals, sea lions, and walruses). Constantine (1982) failed to find evidence for transfer of a matching rule in any of 4 harbor seals (Phoca vitulina) or in a gray seal (Halichoerus grypus). Training was limited to a single matching problem: a black square versus a white square. After successful matching was attained with this pair, a single transfer test was given, in which samples were white stripes on a black background, presented in either a vertical or a horizontal orientation. There was no evidence of immediate transfer or of any savings effect. One problem with Constantine's procedure is that the pinnipeds may have learned to match on the basis of the brightness differences of the original training stimuli. For the transfer problem, brightness was equated and orientation was now the relevant dimension, resulting in an interdimensional transfer problem. Interdimensional transfer is known to be difficult for monkeys 
that otherwise show ready transfer (D'Amato \& Colombo, 1989; D'Amato et al., 1985), and the same is likely true of pinnipeds. Hence, Constantine's (1982) pinnipeds may in fact have experienced some negative transfer. Constantine was eventually able to train matching of the vertical and horizontal stripes, but she gave no further transfer tests. It is unclear, therefore, whether the failure of the pinnipeds occurred because of the difficulty of interdimensional transfer or because only a stimulus-specific matching rule, rather than a general matching rule, was learned during training.

A failure to find transfer of matching in pinnipeds seems inconsistent with other concept-learning data on these species. Pinnipeds are relatively large-brained, social mammals; they can easily be trained to perform complex behaviors in marine parks and circuses. They have good visual acuity in air and underwater (Schusterman, 1972). In several laboratory studies of the California sea lion (Zalophus californianus) (reviewed in Schusterman, 1968) that were done with visual stimuli, including twodimensional patterns, rapid learning-set formation and successful serial reversal learning have been reported. These results indicate an ability to develop general concepts either at the stimulus level (in which one particular object is the discriminative cue) or at the dimension level (in which one particular feature, such as brightness, is the discriminative cue). In other studies, sea lions have learned to respond to multigesture sign sequences of a trainer's arms and hands, in which individual gestures refer to particular objects, actions, or properties and the ordering of the gestures, as well as the particular gestures themselves, conveys meaning (Schusterman \& Krieger, 1984, 1986; cf. Herman, Richards, \& Wolz, 1984). Given the results of these several studies, we might expect a generalized MTS capability in sea lions, inasmuch as some of the necessary skills for generalized matching have already been demonstrated in this species; these include some understanding of identity based on physical characteristics, and an ability to generalize a rule to new instances of the training task. A major goal in the present study was therefore to reexamine the ability of a pinniped-in this case, a sea lion-to form an abstract (generalized) concept of matching. We attempted to avoid the difficulties of Constantine's (1982) procedures by using three-dimensional real-world objects as stimuli, thereby minimizing the probability that the sea lion would focus on any single dimension, and by giving multiple transfer tests. Although we used only a single sea lion subject in our study, this need not be a limitation in deciding on a species' capabilities. As was argued by Wright et al. (1988) in defense of small-sample studies of MTS in pigeons: "Do pigeons have the capacity to learn an abstract concept such as matching-to-sample? In the extreme case, a positive finding from only one subject can answer this question"' (p. 443).

MTS tasks are also useful for the exploration of shortterm working memory capabilities of animals. By varying the time between the removal of the sample stimulus and the presentation of the comparison stimuli, one can assess an animal's ability to form and maintain a representation of the sample stimulus over time and examine how this ability changes as a function of selected variables. In studies of delayed matching-to-sample (DMTS), the short-term memory of dolphins (Herman, 1975; Herman \& Gordon, 1974; Herman ei al., 1989) and of nonhuman primates (D'Amato, 1973; D'Amato \& Worsham, 1972; Jarrard \& Moise, 1971) remains above chance levels after delays of $1 \mathrm{~min}$ or longer after removal of the sample. In contrast, memory for a sample stimulus in pigeons may fall to chance levels after postsample delays of $10 \mathrm{sec}$ or less (see, e.g. , Berryman, Cumming, \& Nevin, 1963; D'Amato \& Salmon, 1984; Grant, 1976; Roberts, 1972; Smith, 1967). Only in an extreme case of over 17,000 DMTS training trials and the use of relatively long sampleexposure durations has short-term memory of the pigeon been extended to as long as 1 min (Grant, 1981).

Constantine (1982) did not conduct DMTS tests with her pinniped subjects. A brief report by Hardenbergh, Schusterman, and Krieger (1987) on memory for objects referred to symbolically suggests that retention declines to chance levels after delays of only 10-15 sec, which is considerably shorter than the values reported for dolphins or nonhuman primates in DMTS tasks, or for a dolphin in similar tests of memory for objects referred to symbolically (Herman et al., 1984).

In summary, the present study, in addition to being a test for a generalized matching capability, is the first test of DMTS in a pinniped. The study thus enlarges the set of relatively large-brained, social mammals examined for matching abilities and short-term memory, and it provides new information on the conceptual and representational abilities of the California sea lion.

\section{EXPERIMENT 1}

In Experiment 1, we tested the ability of a sea lion to learn an identity matching task, using visual materials, and to transfer the matching rule to new visual items or to new pairings of visual items.

\section{Method}

\section{Subject, Apparatus, and Stimuli}

The subject was a juvenile female California sea lion named $\mathrm{Hu}$ apala, approximately 3 years old at the start of this study. She was a test-naive animal born and raised at the Sea Life Park marine animal facility in Hawaii. Huapala was maintained together with a second California sea lion named Kepa in an outdoor enclosure consisting of a rectangular sea-water pool $5.5 \mathrm{~m}$ wide by $4.3 \mathrm{~m}$ long surrounded by a concrete deck area. Both sea lions had free access at all times to the wet and dry areas. Huapala received $8 \mathrm{lb}$ $(3.6 \mathrm{~kg}$ ) of cut fish (herring and smelt) daily as rewards during her training and testing sessions. Daily sessions were conducted in one end of the sea-water pool. Before each session with Huapala, Kepa was moved to an adjacent holding pen.

The wood and Plexiglas apparatus used to display the sample and comparison objects was positioned at the edge of the pool deck facing the water (Figure 1). The apparatus, which was $1.7 \mathrm{~m}$ long and $.9 \mathrm{~m}$ high, consisted of three compartments. The center compart- 


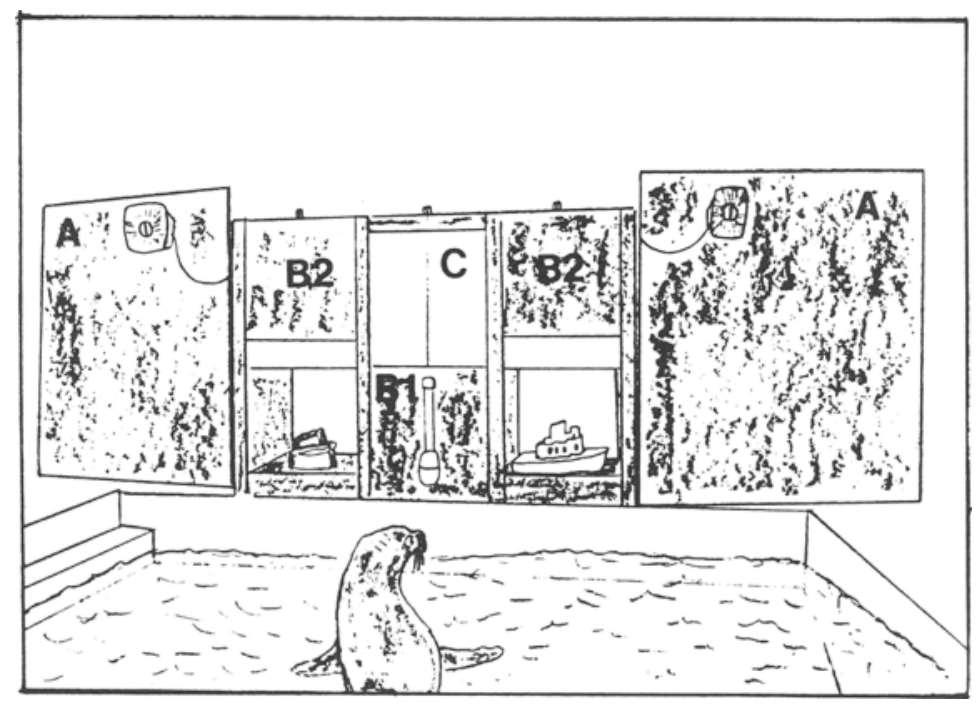

Figure 1. Artist's drawing of the sea lion training pool and matching-to-sample testing apparatus, showing side panels with affixed speakers $(A)$, movable shutters used to display sample and comparison objects (B1 and B2, respectively), and translucent white Plexiglas appearing above each lowered shutter and also at the rear of each compartment $(C)$. The center buoy is attached to B1. The objects shown are the teapot and tugboat.

ment contained the sample object, and each side compartment contained one of the two comparison objects. The objects were placed on the floor of the compartments. Movable shutters in front of each compartment obscured the sea lion's view of the objects. A pulley system allowed the shutters to be raised or lowered from a remote location by the experimenter. A small styrofoam buoy was attached to the center shutter. Huapala pressed this buoy to begin a trial.

The sea lion's view behind the apparatus was obscured by side panels and by a white acrylic sheet above the lowered shutters. The translucent acrylic sheet, the open top of the apparatus, and the shutters, when open, allowed daylight to enter the compartments and illuminate the objects. An Apple II computer generated the sound that was used to signal a correct response. The sound was presented through speakers mounted on the apparatus and controlled by a handheld switch. Hand-held stopwatches were used to time trial events.

The training and transfer stimuli were 12 ordinary "junk" objects: a plastic white jug, a green plastic airplane, a red plastic colander, a vinyl soccer ball, a white rubber boat bumper, a brown and black slipper, a yellow and white plastic lunchbox, a silver teapot, an orange traffic cone, a blue and yellow plastic toy tugboat, a yellow plastic duck, and a square concrete cinder block. They were chosen to be visually distinct from each other as judged by the experimenters, and to fit readily within the confines of the apparatus. The first 3 objects were used during training of the matching task, and the remainder during the transfer tests.

\section{Procedure}

Training. Prior to the introduction of the apparatus and the MTS procedure, Huapala was habituated to eating cut fish (to increase the number of rewards that could be delivered during a training session), trained to go through a gate on command (to separate her from Kepa), and trained to touch objects with her nose when given the appropriate gestural command. In addition, a $640-\mathrm{Hz}$ pure tone was established as a conditioned reinforcer by first pairing it with the presentation of a fish reward and then inserting a short delay between the sound and the fish. This entire pretraining took approximately 8 weeks.
A two-alternative MTS procedure was used in which the sample object was displayed in the center compartment and the comparison objects were displayed in the side compartments. Huapala was trained to touch the buoy placed on the center shutter to begin a trial, to touch the sample object after the center shutter was raised, and finally to touch one of the two comparison objects to indicate her choice after the side shutters were raised.

An intertrial interval (ITI) of $30 \mathrm{sec}$ was used throughout the experiment. Before the start of each trial, the sample and comparison objects were placed in the appropriate compartments from behind the apparatus according to a preplanned schedule balanced for sample object and for location (left or right) of the matching comparison object $(\mathrm{S}+)$. The object used as the sample was governed by a preplanned pseudorandom schedule under the restrictions that the same object not be used as the sample more than three times successively, and the $S+$ not appear in the same location for more than three successive trials.

To begin a trial, the experimenter, standing at the rear of the apparatus, vocally called to Huapala as a signal for her to approach the apparatus; when Huapala approached, the experimenter sat down and remained hidden from Huapala's view throughout the trial. Huapala's responses could not be observed by the experimenter. An assistant, located outside the pen, observed Huapala and instructed the experimenter appropriately - that is, to raise the center shutter, lower it, or raise the side shutters. The assistant also indicated whether or not Huapala's choice of comparison object was correct.

When the assistant observed that Huapala had touched her nose to the buoy attached to the center shutter, she vocally instructed the experimenter to raise the center shutter to expose the sample object. The center shutter was lowered after Huapala's first nosetouch of the sample object after a 5-sec exposure interval had elapsed (FI 5-sec schedule). The two side shutters were then raised simultaneously, revealing the comparison objects. Formally, this constituted a zero-delayed MTS procedure. A response to a comparison object was defined as a touch of the object with the nose. This straightforward response was easily judged by the assistant, who vocally signaled the experimenter when a response was made and 
indicated whether it was correct or not. A correct choice produced the conditioned-reinforcer tone. The shutters were then lowered and the experimenter exited from behind the apparatus and fed the sea lion one piece of cut fish. If Huapala's choice was incorrect, the shutters were lowered, the conditioned reinforcer tone and fish reward were omitted, and the experimenter remained behind the apparatus.

If Huapala failed to touch the center buoy within $1 \mathrm{~min}$ after being signaled, a 3-min time-out period was given, during which all shutters were closed and any contact with the experimenter was excluded. This period was increased to $10 \mathrm{~min}$ if Huapala again refused to approach. The session was terminated after the third successive failure to approach the buoy. Huapala was then fed half of her daily ration $1 \mathrm{~h}$ later. Also, if Huapala did not touch the sample object within approximately $25 \mathrm{sec}$ after the center shutter was raised, the shutter was lowered, the ITI was restarted, and afterwards the same trial was repeated.

The jug, airplane, and colander were used as training stimuli during the initial acquisition phase of the matching experiment. Standard training sessions consisted of 24 noncorrection trials. Fewer trials were run if the session was terminated prematurely because of Huapala's reluctance to begin or continue trials. During this initial phase of training, the sea lion developed a strong aversion to the colander, refusing to approach it. Consequently, the colander was removed from the stimulus set, and training was continued with the jug and airplane alone. Also, the reward for a correct response was changed from one piece of herring to three pieces of herring, in an attempt to increase motivation. Training with the jug and airplane was continued until a criterion of $90 \%$ correct responses or better was reached over two consecutive sessions.

Transfer tests. Testing for transfer began immediately after the training phase was successfully completed. The first two tests constituted partial transfers, in which a new object, the white ball, was paired with each of the final two objects used in training. In Transfer Set 1, the ball and the airplane were paired; in Transfer Set 2, the ball and the jug. Each object of a pair served as the sample equally often, and all of the trial constraints used during training continued to be used during transfer testing. Testing with each of these first two transfer pairs continued until the criterion of $90 \%$ correct responses or better over two consecutive sessions was met.

In four subsequent transfer tests, novel pairs of objects were presented: in Transfer Set 3, the buoy and slipper; in Transfer Set 4, the teapot and lunchbox; in Transfer Set 5, the cone and tugboat; and in Transfer Set 6, the duck and cinder block. So that these scheduled transfer tests could be completed within the time available for the experiment, the criterion level was lowered to $80 \%$ correct responses or better on two consecutive sessions of Transfer Sets 5 and 6. The objects used in Transfer Sets 3, 4, and 5 had not been seen by Huapala prior to their use on the first trial of a transfer test. Because of Huapala's initial reluctance to approach these new objects, a preexposure procedure was used for Transfer Set 6 . This consisted of 14 trials of exposure of the duck or block in the center compartment, with each object exposed seven times. The ordering of objects followed a pseudorandom schedule. Upon exposure of an object, Huapala was gesturally signaled by the experimenter to approach and contact the object with her nose. Testing for transfer began immediately after completion of these 14 preexposure trials.

\section{Training}

\section{Results and Discussion}

Huapala was given a total of 772 training trials. The first 503 (Sessions 1-24) included the use of all three training objects-jug, airplane, and colander-but the performance criterion was not reached. During these 24 sessions, Huapala showed strong side biases, object preferences, and object avoidance. Correction trials were sometimes used to counteract inappropriate choice strategies. However, this usually resulted in biases changing over the course of a session or over groups of sessions, without a noticeable increase in accuracy of response. Accuracy differed significantly among the three samples: $66.4 \%, 42.9 \%$, and $27.2 \%$ correct responses, respectively, for the airplane, jug, and colander as samples [ $\chi^{2}(2)$ $=48.4, p<.001]$. The noticeably poor performance with the colander as the sample reflects Huapala's tendency to avoid this object, a tendency that increased as sessions progressed.

The colander was eliminated from the object pool on Session 25 and thereafter, and an additional 269 trials were given with the airplane and jug alone. This resulted in the general improvement in performance shown in Figure 2. Although there was a steep decline in performance during Session 26, resulting in the early termination of this session, this was followed by a nearly steady increase in performance through to Session 36 when the $90 \%$ performance criterion over two successive sessions was reached. Huapala responded correctly on 23 of 24 trials during Session 35 and on all 24 trials during Session 36 .

\section{Transfer Testing}

Acquisition. Figure 3 shows the percentage of correct responses for each transfer test at each testing session until the performance criterion was reached. All sessions consisted of 24 trials, except for three that were terminated early because of object avoidance by Huapala. Although the first four problems had a $90 \%$ criterion and the last two an $80 \%$ criterion, to simplify comparisons both the $80 \%$ and $90 \%$ correct levels are shown for all six problems.

If we examine the in-common $80 \%$ criterion (over two successive sessions) for all problems, it can be seen that successful transfer was attained in the minimum of two sessions in one case (Set 2), in three sessions in two cases (Sets 1 and 6), in four sessions in two cases (Sets 4 and 5 ), and in six in the remaining case (Set 3). For Set 3, the first wholly novel pairing, Huapala was correct on

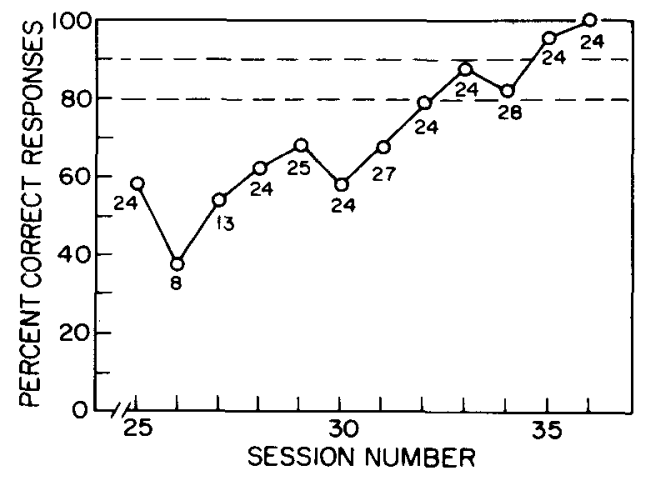

Figure 2. Acquisition of the matching rule with the training objects, plane and jug. The $80 \%$ and $90 \%$ criteria are shown. The number of trials given in each session is shown above each data point. 


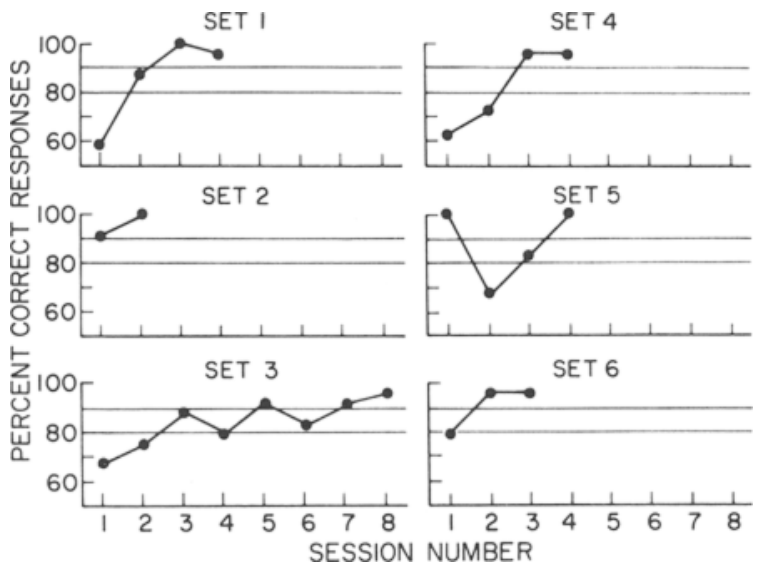

Figure 3. Matching accuracy on Transfer Sets 1-6 until the criterion was reached: $90 \%$ correct responses over two consecutive sessions for Sets 1-4 and $80 \%$ correct responses over two consecutive sessions for Sets 5 and 6 . Each data point is based on 24 trials, except in Session 2 of Set 4 (18 trials) and Sessions 1 and 2 of Set 5 ( 2 and 3 trials, respectively).

$87.5 \%$ of the 24 trials of Session 3 and on $79.2 \%$ of the 24 trials of Session 4 , barely missing the $80 \%$ criterion at that point. Set 3 was continued for eight sessions altogether in order to meet the criterion of $90 \%$ correct responses in effect at that time. Thus, in summary, the $80 \%$ performance criterion was reached, or closely approximated, relatively quickly in all transfer sets.

Errors to criterion. Figure 4A shows the number of errors to reach the $80 \%$ and $90 \%$ criteria for the training problem consisting of the jug and plane alone and the two partial transfers: Transfer Set 2-ball and plane-and
Transfer Set 2-ball and jug. The number of errors for the training problem are those occurring only after the colander was removed from the set-that is, for Sessions 25 through 36 (Figure 2), which included only the jug and plane. If the results for Training Sessions 1-24 were included in the error totals, the number of errors to criterion would increase greatly, producing a spuriously large error value against which subsequent transfer performance would be evaluated.

Figure 4A reveals that the number of errors to either criterion were few for the partial transfer sets (a maximum of 13 errors for Set 1, and 2 errors for Set 2), both in absolute terms and in relative terms when compared with the large number of errors observed in the training set (69 and 70 errors, respectively, for the $80 \%$ and $90 \%$ criteria).

Figure 4B shows the number of errors to criterion for Transfer Sets 3-6, all of which had novel pairs of objects. A rise in errors to criterion occurred during the first novel transfer set, relative to that obtained with the immediately preceding partial transfer set, although the number of errors (a maximum of 31) was still well below that observed with the training set. Thereafter, during the final three novel transfer sets, errors decreased substantially relative to the first novel transfer set or relative to the training set. For Transfer Set 4, the maximum number of errors was 16; for Transfer Set 5, it was 5; and for Transfer Set 6 , it was 7 .

The generally improved level of performance over successive novel transfer tests might be explained by the increased familiarity of the sea lion with the task, or by other general-learning effects that do not reflect concept formation. The data suggest that task familiarity had likely exerted its maximum benefit before the novel transfer tests

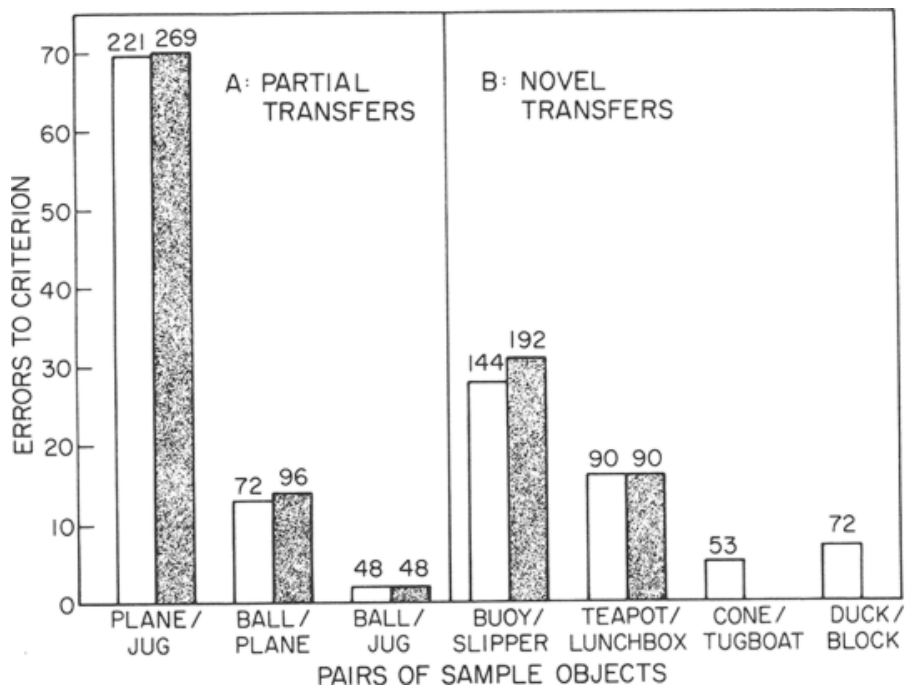

Figure 4. (A) The number of errors to criterion for the final training set (plane and jug) and for the two partial transfer tests. (B) The number of errors to criterion for each of the novel transfer sets. The number of trials to criterion is shown above each bar; open bars indicate the $80 \%$ criterion, filled bars the $90 \%$ criterion. 
were given. First-as we noted earlier-during the final two sessions of the training problem (see Figure 2), performance had reached a near-ceiling or ceiling level ( $96 \%$ and $100 \%$ correct responses, respectively, during Sessions 35 and 36). Second, during the final partial transfer test, in which the ball and jug, by then familiar objects to Huapala, were compared, performance again approached a ceiling level: The problem was completed in the minimum of two sessions (48 trials) with only two errors occurring throughout (Figure 3 ). Had task familiarity still been accruing, ceiling level performance during the final two sessions of the training problem or the first two sessions of the second partial transfer test would not have been expected. Instead, what seems to characterize the difference between the end of the training problem or the second partial transfer test and the subsequent novel transfer problems is object familiarity, not task familiarity.

Another general learning factor sometimes implicated in improved performance across multiple exemplars of a problem is enhanced attention to relevant stimulus dimensions leading to rapid stimulus-specific learning. Although this factor cannot be dismissed entirely in the present tests, the types of stimuli we used tended to guard against any particular dimension's being singled out as consistently relevant. Consider, for example, the substantial differences in appearance between tugboat, cone, block, and duck, the objects used in the final two transfer tests. It would be difficult to identify a common relevant dimension among these objects that would facilitate stimulus-specific learning. D'Amato et al. (1985) have in fact cautioned against using stimuli restricted to some elementary dimension (e.g., wavelength) in training and transfer tests of matching, because of the uncertainty of attributing positive results to concept development or to stimulus generalization. Hence, the results of the novel transfer tests may be best viewed as representative of $\mathrm{Hu}$ apala's ability to apply the identity rule to new objects.

As was noted earlier, and as has been described by others (e.g., D'Amato et al., 1985; Zentall \& Hogan, 1978), the potential for rapid transfer can be masked by phobic responses to new objects. Huapala, like the monkeys of D'Amato et al. and the pigeons of Zentall and Hogan, showed strong neophobic responses during most of the transfer tests. During the first session of Transfer Set 1, Huapala refused to respond to the new ball as the sample during 13 exposures of that object before finally responding reliably. Transfer Sets 3, 4, and 5 required, respectively, 13, 17, and 20 exposures of sample objects before reliable responding to the sample was obtained. The 17 exposures of Set 4 and the 20 exposures of Set 5 were each given over two sessions, because of severe behavioral disruption, which forced an early termination of the second session of Set 4 and both the first and the second sessions of Set 5. Only Sets 2 and 6 failed to elicit strong object avoidance: Set 2 contained no novel objects and Set 6 was the only set with preexposure of sample objects. These findings, plus the character of Huapala's responses, strongly suggest neophobia. Her responses to new objects had a characteristic pattern: Typically, she jumped back from the center shutter when a new sample object was first revealed, refused to approach the apparatus again for nearly $1 \mathrm{~min}$, and often paced from one side of her pool to the other during the ITI. Such responses generally abated by the end of the first session, or by the end of the first 24 trials if these spanned more than one session.

Because of the neophobic responses of their cebus monkeys, D'Amato et al. (1985) relied on performance during Sessions 2, 3, and 4 for evaluation of transfer, rather than on Session 1. These authors also used a performance criterion for determining transfer $\mathbf{( 7 0 . 8 \%}$ correct responses or better during Sessions 2, 3, and 4) that was less stringent than the criterion of $80 \%$ (or $90 \%$ ) correct responses over two successive sessions adopted here. Only 4 of 8 monkeys passed the first transfer test by D'Amato et al.'s criterion. Three of the 4 that failed the first test were given a second transfer test, and 2 passed. How might the sea lion's performance compare with that of the cebus monkeys if we apply D'Amato et al.'s criterion to the novel transfer tests? Apparently very well, inasmuch as this criterion was met during Sessions 2, 3, and 4 of Transfer Sets 3 and 4 , and during all sessions of Transfer Set 6. Only Transfer Set 5 appears to fail: Session 2 performance fell below $70.8 \%$, although Sessions 1,3 , and 4 all exceeded that value. The first two sessions of Set 5 were highly abbreviated, however, consisting of only 2 and 3 completed trials, respectively. If we instead combine the results of Sessions 1, 2, and 3, D'Amato's criterion is met for these combined sessions and for the one remaining session that followed. These comparisons with the results of D'Amato et al. must be taken with some caution, however, because the twodimensional forms used by D'Amato et al. were likely more difficult discriminations than were the threedimensional objects used in this study.

\section{Rapidity of Transfer}

The most stringent measure of rapidity of transfer we used was based on Huapala's performance during the first four unique trials of each transfer problem. If we designate the two samples of a transfer test as $A$ and $B$, the four possible unique trial types are: Sample $=A$, match on left; Sample = A, match on right; Sample $=$ B, match on left; Sample = B, match on right. These four unique trial types were the first four trials given to the sea lion in each transfer test, except in Transfer Set 5. The strong neophobic responses during the first and second sessions of Set 5 resulted in the early termination of these sessions and precluded our carrying out the trial sequences as planned.

Thus, for each transfer set, the number of correct responses over these four unique trials could range from zero to four. The results of this measure were the following: For the two partial transfer sets (Sets 1-2), there were, respectively, 2 and 3 correct responses (for $n=$ 8 trials, $p=.36$ ); for the four novel transfers (Sets 3-6), 
the numbers of correct responses were, respectively, 3 , 2,3 , and 2 (for $n=16$ trials, $p=.23$ ); and for the combined sets, there were 15 correct responses altogether ( $n=24$ trials, $p=.15$ ). This most stringent measure thus fails to give evidence of immediate transfer.

Rapidity, rather than immediacy of transfer, may be measured by comparing the results of the novel transfer tests with some baseline measure of matching performance. A conservative baseline measure, one that guards against overinterpreting Huapala's transfer performance, would be performance on the second partial transfer test. We noted that this test was passed rapidly, in the minimum possible 48 trials, with but two errors occurring, and that the stimuli used for this test were familiar to $\mathrm{Hu}$ apala through their use during training or during Partial Transfer Test 1. Performance on this second partial transfer test can therefore be used as a standard against which performance on the first 48 trials of each of the four novel transfer tests is compared. The first novel transfer test (Set 3 ) yielded 14 errors in the first 48 trials, the second (Set 4) 15 errors, the third (Set 5) 5 errors, and the fourth (Set 6) 6 errors. Both chi-square and a test for the equality of two percentages based on arcsine transformations of percentages (Sokol \& Rohlf, 1969, p. 607) were used to evaluate the differences between the baseline performance and those for each of the transfer tests. The difference between performance on baseline and on each of the first two novel transfers was significant $(p<.02)$ by both statistical tests, but the differences between baseline and the last two transfers was not $(p>.25)$. Thus, the last two novel transfers were not learned significantly more slowly than was the baseline test, thereby providing evidence for rapid transfer of the matching concept.

In summary, the results suggest that the sea lion developed a concept of matching and was able to apply it relatively quickly (but not immediately) to novel transfer problems, especially the last two given. A limiting condition on performance throughout most of the transfer tests consisted of the phobic response of Huapala to novel stimuli.

\section{EXPERIMENT 2}

The sea lion's memory for the sample object shown at each trial was tested by introducing delays between removal of the sample object and the presentation of the comparison objects. During Part A of the experiment, delays ranged from 3 to $28 \mathrm{sec}$, and during Part B, they ranged from 3 to $58 \mathrm{sec}$.

\section{Method}

\section{Subject, Apparatus, and Sample Objects}

Huapala continued as the subject of Experiment 2. She was tested in the same pen as in Experiment 1, using the same apparatus. Three objects-tugboat, teapot, and slipper-were chosen from Experiment 1 and used as sample stimuli during these DMTS tests. Three objects, rather than two, were used as samples to help reduce the effects of proactive interference on accuracy of responding during the current trial. The events or responses of the prior trial have been shown in several studies to interfere with choice accuracy on the current trial, especially if only one of two sample events can occur at each trial (see, e.g., D'Amato, 1973; Herman, 1975; Herman \& Thompson, 1982; Roitblat, 1980; Roitblat \& Scopatz, 1983; Wright, Urcuioli, \& Sands, 1986).

\section{Procedure}

Pretraining. Prior to the start of formal DMTS testing, Huapala was given a brief period of pretraining with delays to accustom her to a waiting period after the exposure of the sample object ended and before the comparison objects were presented. A total of five sessions (120 trials) were given in which the block, teapot, and tugboat were samples. Short delays of $6 \mathrm{sec}$ or less were mainly used, interspersed occasionally with longer delays of 14 or $30 \mathrm{sec}$. During this pretraining phase, Huapala showed a strong preference for the block. Accordingly, we substituted the slipper for the block and trained her for an additional two sessions ( 48 trials), using the new set of three objects (slipper, teapot, and tugboat). Delays ranged from 3 to $10 \mathrm{sec}$. Huapala showed no strong preferences or aversions with this new set, and formal DMTS testing (Part A) began with the next session.

Part A. Each trial consisted of, sequentially: (1) a display of one of the three sample objects, according to the same presentation methods and with the same duration as in Experiment 1 ; (2) the occurrence of a predetermined delay interval of $3,6,10,15,21$, or $28 \mathrm{sec}$ in a mixed and balanced sequence within sessions; and (3) the display of two comparison objects, one of which matched the sample. During the longer delays of the pretraining phase, $\mathrm{Hu}-$ apala at times moved rapidly back and forth between the two comparison objects after their exposure, making the judgment of her choice of comparison object difficult. The response criterion was therefore altered slightly for Part A to require that Huapala remain stationary in front of one or the other of the two comparison objects for $3 \mathrm{sec}$ before a judgment of a response was made. Additionally, a "blind" observer, who was unaware of which comparison object was correct, announced the sea lion's choice. This control guarded against any experimenter bias in judging responses. A minimum ITI of $45 \mathrm{sec}$ was used. Several of the studies on proactive interference cited earlier have shown that longer ITIs (ca. 15-30 sec or greater) help reduce the interfering effects of prior trials. The actual length of the ITI that we used averaged $54.0 \mathrm{sec}(S E=$ $6.67 \mathrm{sec}$ ) and depended in part on how soon the sea lion returned to the apparatus after being signaled by the experimenter to press the center buoy.

Each session consisted of 24 trials ordered pseudorandomly with no sample or distractor object and no side compartment for the S+ repeated more than three times in succession. Since there were six unique pairwise permutations of the three objects, two possible positions for the $S+$ object (left or right compartment), and six delays, a complete balanced replication required 72 trials $(6 \times 2 \times$ $6)$, or three sessions. A total of six replications (18 sessions) were completed in Part A.

During each daily session, each of the six permutations of sample and distractor appeared four times, with the $S+$ appearing twice in the left compartment and twice in the right. Also, each of the six delays appeared four times during a session. The association of particular delays with particular samples was partially balanced, insofar as was possible within the $\mathbf{2 4}$ trials given. Complete balancing occurred over the course of three sessions.

Part B. Part B was conducted exactly as Part A, with the exception that the six delays were set to $3,12,22,33,45$, and 58 sec. Because these delays were longer, on the average, than those used in Part A, only 18 trials were given per session. This decreased number of trials maintained the session length at approximately the same duration as that of the sessions of Part A. Complete balancing of variables therefore required four sessions rather than the three 
used in Part A. As in Part A, a total of six replications (24 sessions) were completed. The ITI averaged $56.1 \mathrm{sec}(S E=13.43 \mathrm{sec})$.

\section{Results and Discussion}

Figure 5 depicts the sea lion's response accuracy as a function of the delay after removal of the sample object, for both Part A and Part B of the experiment. High levels of performance were achieved: accuracy was above $90 \%$ correct through to delays of $6 \mathrm{sec}$ and at or above $78 \%$ correct through to 45 -sec delays; it finally declined to $69 \%$ at the final 58-sec delay. All values were significantly greater than chance, using a protected one-tailed $t$ test $[t(216) \geq 1.9, p<.05]$. Furthermore, these retention levels are only somewhat below levels reported for dolphins (Herman et al., 1989) or monkeys (D'Amato, 1973) tested on visual DMTS.

Analyses of variance were applied to the data of Part A and, separately, to Part B. Of interest were the effects on DMTS performance of different samples (because of potential preferences or avoidances) and the effects of the successive replications (i.e., the effects of DMTS practice). To increase the power of the test, additional variables were entered into the analysis: specifically, delays, the position of $\mathbf{S}+$ (in the left or right compartment), and the particular distractor object $(\mathrm{S}-$ ) used on the matching trials. Since there were three objects, each $S+$ could be paired with either of the two remaining comparison (distractor) objects. To test for practice effects, the six replications were grouped into three blocks of two successive replications. The three blocks were each based on 144 trials and represent, respectively, early, middle, and late stages of practice.

Each of the 432 trials given the sea lion during each part of the experiment was an analyzed observation. The dependent variable was whether the trial response was correct or incorrect. Analyses of this type are discussed in Overall (1980) and Cohen and Cohen (1983). The effects tested accounted for $67 \%$ of the variance in Part $A$, and for $64 \%$ in Part B. In both parts, all main effects were significant. In Part $\mathrm{A}$, the difference among samples was highly significant $[F(2,216)=29.6, p<.0001]$, primar-

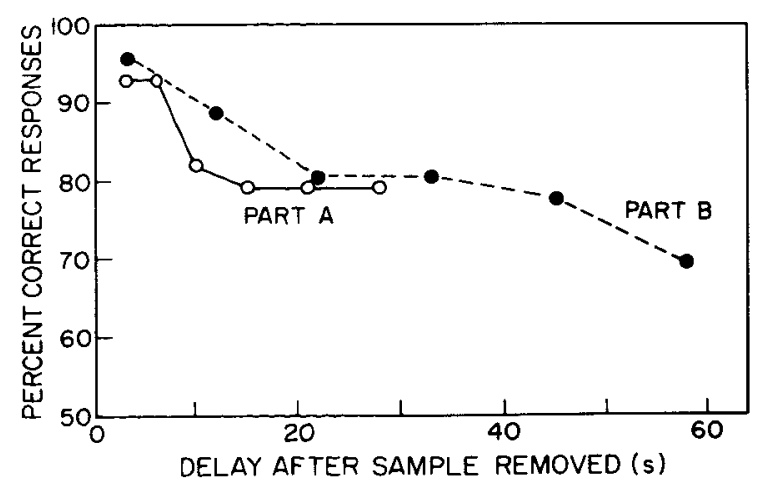

Figure 5. Matching accuracy during delayed MTS as a function of delays used in Part A $(3,6,10,15,21$, and 28 sec) and Part B $(3,12,22,33,45$, and $58 \mathrm{sec})$.

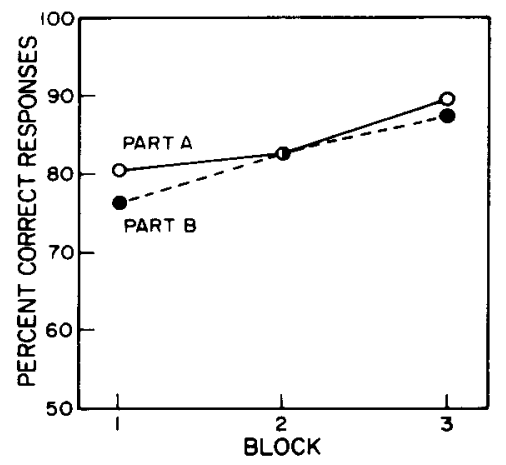

Figure 6. Delayed MTS accuracy as a function of practice (blocks of 144 trials) during Parts $A$ and $B$.

ily reflecting the weaker performance with the slipper (68.8\% correct) than with the tugboat $(\mathbf{9 2 . 4 \% )}$ ) or teapot $(91.7 \%)$. Subsequent Scheffé tests revealed that the difference in performance with each of the latter two objects as opposed to the slipper was significant $[t(216) \geq 6.56$, $p<.001]$. In Part B, which contained longer delays, these performance differences were reduced, although the overall effect of samples was still significant $[F(2,216)$ $=6.0, p<.005]$. Performance with the slipper improved to $79.9 \%$, while performance with the tugboat (77.1\%) was reduced from that in Part A. Performance with the teapot $(89.6 \%)$ remained nearly the same and significantly exceeded that with each of the two other objects $[t(216) \geq 2.56, p<.05]$. In neither Part $A$ nor Part B was there a significant interaction between samples and delay intervals. These results show, not surprisingly, that performance in DMTS tests can vary among objects, perhaps because of object biases, or differences in the perceptual saliency or memorability of the objects. However, the influence or importance of these variables apparently may change with practice.

In both Part $A$ and Part B, overall performance improved over the three successive blocks, as is shown in Figure $6[F(2,216) \geq 3.6, p<.05]$. In each case, the only significant contrast occurred between Block 1 and Block $3[t(216) \geq 2.58, p<.01]$. For Part B only, the interaction of sample and blocks was significant $[F(4,216)$ $=2.9, p<.05$ ], resulting from relatively large differences in performance with the three objects during Block 1, relatively small differences during Block 2, and virtually no differences during Block 3 . These results are consistent with data from other studies (e.g., D'Amato, 1973; Herman \& Gordon, 1974), which show that DMTS performance of some species may improve with experience with DMTS tests, perhaps because of increased attention to the relevant stimulus dimensions, increased familiarity with the requirements of the matching task, or improved strategies for remembering.

\section{GENERAL DISCUSSION}

The results demonstrate that a sea lion can learn a concept of identity, evidenced by its ability to apply the 
matching rule to new problems. The strongest test of transfer of the rule-immediate matching, measured by errorless or near-errorless performance on the first four unique trials of each new matching problem-was not passed, but this was interpreted as at least partly the result of timidity when the sea lion was confronted with new objects. The cebus monkeys tested for transfer of a matching rule by D'Amato et al. (1985) experienced similar phobic reactions to novel objects, and this reaction has been observed in other studies with monkeys (Jackson \& Pegram, 1970) and pigeons (Zentall \& Hogan, 1979) as well. Our other tests for transfer showed that the performance criterion ( $80 \%$ or $90 \%$ correct responses over two successive sessions) was reached or closely approximated in two to four transfer sessions for both partial transfer tests as well as for all four of the novel transfer tests; that errors to criterion tended to be reduced across the successive novel transfer tests and were as few as five during the final two tests; and that performance on the last two novel transfers was not significantly different from the near-ceiling level baseline performance on the second of the two partial transfer tests $(96 \%$ correct responses in the first 48 trials). We were able to exclude increased task familiarity as contributing to the levels of performance attained on the novel transfer tests because of the near-ceiling levels of performance achieved in the final two training sessions and on the final partial transfer test, both of which preceded the start of the novel transfers. We could not completely exclude improvement in the rate of learning stimulus-specific associations as a contributing agent to the heightened performance observed during the final two novel transfers. We considered, however, that any contribution would be limited by the disparate appearance of the stimuli used across transfer tests that would seem to protect against solutions based on simple stimulus generalization.

As Oden et al. (1988) have stressed, immediate (i.e., first-trial) transfer of the matching rule from a limited set of training stimuli to novel stimuli is a rare finding. Chimpanzees (Nissen et al., 1948; Oden et al., 1988) and dolphins (Herman et al., 1989; Hunter, 1988) have shown this capability, giving evidence that they construe the identity relationship from the beginning in the broad sense of a concept of same/different, applicable to any stimulus material (Oden et al., 1988). In contrast, some animals may narrowly attend to the identity relationships among the particular stimulus pairs used, such that the matching concept develops rather slowly, if at all, as more problems are given. The latter description has characterized the performance of pigeons tested on identity problems (e.g., Wright et al., 1988).

In other studies in which generalized matching has been obtained (e.g., D'Amato et al., 1985), the strong neophobic reactions of naive animals have led researchers to use alternative measures of transfer (e.g., performance on sessions after the neophobic responses have diminished). The young chimpanzees studied by Oden et al. (1988) were also naive, but prior to the start of MTS testing they were exposed to a variety of objects, including those used at the later transfer tests. The chimpanzees were allowed free play with the objects and were also rewarded for their initial choice in paired-comparison forced-choice tests. Objects provoking strong attraction or avoidance responses were not used in the subsequent matching tests. Under these procedures, the chimpanzees did not exhibit neophobic responses, allowing an unbiased estimate of first-trial transfer capability. In our study, preexposure was used only for the final transfer problem comparing duck and block, but our procedure was much less extensive than that used by Oden et al., consisting of only seven contact trials with each object that appeared in the center compartment. Nevertheless, the procedure did seem to decrease neophobia. In contrast with her performance on the three preceding novel transfers, Huapala never once aborted a trial with the duck and block, nor did she hesitate to approach and touch either object when it was the sample (i.e., in the center compartment). She did, however, show initial strong hesitancy in approaching the side compartment containing the block when it was the $\mathrm{S}+$ alternative. On several occasions, she approached the block correctly but then veered away suddenly and touched the duck. Both errors made during the first four unique trials of the first session were characterized by an avoidance of the side compartment containing the block. Thus, the preexposure condition we used was helpful to some degree, but perhaps because we failed to include preexposure in the side compartments Huapala's habituation was not complete.

A previous study of pinnipeds (Constantine, 1982), in which four harbor seals and a gray seal were the subjects, did not yield evidence for formation of a matching concept. The resolution of those results with our successful findings may reside in the different kinds of stimulus materials and transfer tests applied. First, Constantine used two-dimensional patterns, whereas we used threedimensional real-world objects that may have been perceptually simpler discriminations. Second, Constantine's single transfer test was in effect an interdimensional transfer; the training problem could have been solved (and likely was solved) by attention to brightness differences, but these brightness differences were absent on the transfer problem. Third, Constantine proceeded directly from a single training problem to a transfer test involving novel materials. In contrast, we followed training with two partial transfer problems before testing novel transfers. The question of species differences also remains, but studies by Schusterman $(1968,1972)$ have shown that harbor seals, the major subject group studied by Constantine, are as proficient as sea lions on visual size and area discriminations, as well as in forming an efficient learning set (Harlow, 1949) over a series of pattern discrimination problems. Thus, procedural differences in the MTS studies carried out by Constantine and by us seem the most likely agent leading to the different results obtained.

The present results add to the body of data which show that a variety of animals are able to acquire the matching 
concept, although the rapidity or ease with which the concept emerges and the degree of abstractness of the concept may differ substantially among species. Basically, "matching" is a concept that concerns the identity relationships among stimuli. Other studies have shown that sea lions are also capable of forming concepts that are based on the semantic relationships among objects referenced within an artificial gestural language (Schusterman \& Krieger, 1984; cf. Herman et al., 1984). Using gestures to refer to objects, Hardenbergh et al. (1987) estimated a sea lion's short-term memory (STM) for "named" objects to last $15 \mathrm{sec}$ at the most. The results of the STM tests in the present study indicate that the sea lion subject was able to maintain a representation of a displayed object in memory reliably for almost $1 \mathrm{~min}$, the longest delay tested. Although the details of the Hardenbergh et al. study are not available, the performance differences are probably not due to differences in the type of discrimination used-a conditional discrimination, or conditional matching task, for Hardenbergh et al.'s sea lion, and an identity matching task in the present study. In studies of STM in dolphins (Herman \& Thompson, 1982) and monkeys (D'Amato \& Worsham, 1974), no substantial differences have been found between conditional (symbolic) DMTS and identity DMTS once the conditional relationship has been learned well, as was certainly the case in the Hardenbergh et al. (1987) study.

Under the conditions of the present study, memory for a visual sample remained reliable after delays as long as $58 \mathrm{sec}$, a value not much below levels reported for a dolphin (Herman et al., 1989) or nonhuman primate (D'Amato, 1973). The improvement in the sea lion's memory for the sample over blocks of DMTS tests is consistent with data from monkeys (D'Amato, 1973) and dolphins (Herman \& Gordon, 1974), which show substantial improvements in DMTS performance with increasing experience in the task. Conceptually, the beneficial effect of practice indicates that the ability to retain stimulus information faithfully in memory may depend on enrichments of the representation of that stimulus. Enriched representations may emerge relatively slowly as experience with a stimulus accrues (Roitblat, 1980). In delayed matching studies, the trial-by-trial exposure to the sample stimuli may gradually result in enhanced saliency of the samples, more efficient or more detailed stimulus coding, and heightened attention to and proficiency in the task of remembering.

Finally, Mardon and Herman (1987) recently reported that a California sea lion was able to learn a same/different task in which two arbitrary sounds were used as training stimuli. The sea lion was taught to press a paddle if two sequentially presented sounds were the same and to withhold the response if the two sounds were different. Transfer tests with novel sounds suggested that the sea lion had acquired a generalized same/different rule.

No extraordinary procedures were required for our sea lion subjects to achieve successful transfer (cf. Shyan, Wright, Cook, \& Jitsumori, 1987; Wright, Shyan, \& Jitsu- mori, 1990). In both the present study of visual matching and the Mardon and Herman (1987) study of auditory matching, the animals were apparently able to appreciate relatively quickly the abstract nature of the relationship between different stimuli. Taken together, these results provide a further illustration of modality independence in the execution of complex cognitive tasks by some animals (cf. Herman \& Gordon, 1974; Herman et al., 1989 , for auditory and visual work with dolphins, and Colombo \& D'Amato, 1986; D'Amato, 1973; D'Amato \& Colombo, 1985; D'Amato et al., 1985; Shyan et al., 1987; and Wright et al., 1990, for work with monkeys). The conventional view has been that animals tend to be limited in their ability to solve complex cognitive tasks when task information appears in a "secondary" sensory modality (e.g., Thompson, 1981), a view that seems to be in need of revision if it is intended as a broad generality.

\section{REFERENCES}

Berryman, R., Cumming, W. W., Nevin, J. A. (1963). Acquisition of delayed matching in the pigeon. Journal of the Experimental Analysis of Behavior, 6, 101-107.

COHEN, J., \& COHEN, P. (1983). Applied multiple regression/correlation analysis for the behavioral sciences. Hillsdale, NJ: Erlbaum.

CоLомво, M., \& D'AMAто, M. R. (1986). A comparison of visual and auditory short-term memory in monkeys (Cebus apella). Quarterly Journal of Experimental Psychology, 38B, 425-448.

Constantine, B. J. (1982). An experimental analysis of stimulus control in simple conditional discriminations: A methodological study (Doctoral dissertation, Northeastem University, 1981). Dissernation Abstracts Intemational, 42, 4599B.

D'Aмsтo, M. R. (1971). Sample familiarity and delayed matching in monkeys. Psychonomic Science, 25, 179-180.

D'Aмato, M. R. (1973). Delayed matching and short-term memory in monkeys. In G. H. Bower (Ed.), The psychology of learning and motivation: Advances in research and theory (Vol. 7, pp. 227-269). New York: Academic Press.

D'Aм^ то, M. R., \& CоLомво, M. (1985). Auditory matching-to-sample in monkeys (Cebus apella). Animal Leaming \& Behavior, 13, 375-382.

D'Aмато, M. R., \& CоLомво, M. (1989). On the limits of the matching concept in monkeys (Cebus apella). Journal of the Experimental Analysis of Behavior, 52, 225-236.

D'Amato, M. R., SALmon, D. P. (1984). Cognitive processes in Cebus monkeys. In H. L. Roitblat, T. G. Bever, \& H. S. Terrace (Eds.), Animal cognition (pp. 149-168). Hillsdale, NJ: Erlbaum.

D'Amato, M. R., Salmon, D. P., \& Colombo, M. (1985). Extent and limits of the matching concept in monkeys (Cebus apella). Journal of Experimental Psychology: Animal Behavior Processes, 11, 35-51.

D'Amato, M. R., Worsham, R. W. (1972). Delayed matching in the capuchin monkey with brief sample durations. Learning \& Motivation, 3, 304-312.

D'Amato, M. R., Worsham, R. W. (1974). Retrieval cues and shortterm memory in Capuchin monkeys. Joumal of Comparative \& Physiological Psychology, 86, 274-282.

Grant, D. S. (1976). Effect of sample presentation time on long-delay matching in the pigeon. Learning \& Motivation, 7, 580-590.

Grant, D. S. (1981). Short-term memory in the pigeon. In N. E. Spear \& R. R. Miller (Eds.), Information processes in animals: Memory mechanisms (pp. 227-256). Hillsdale, NJ: Erlbaum.

Hardenbergh, R. A., Schusterman, R. J., * Krieger, K. (1987). Short-term memory in a California sea lion. Poster presented at the Sixth Biennial Conference on the Biology of Marine Mammals, Vancouver, British Columbia, Canada.

HARLOW, H. F. (1949). The formation of learning sets. Psychological Review, 56, 51-65. 
Herman, L. M. (1975). Interference and auditory short-term memory in the bottlenosed dolphin. Animal Learning \& Behavior, 3, 43-48.

Herman, L. M., \& Gordon, J. A. (1974). Auditory delayed matching in the bottlenosed dolphin. Journal of the Experimental Analysis of Behavior, 21, 19-26.

Herman, L. M., Hovancik, J. R., Gory, J. D., \& Bradshaw, G. L. (1989). Generalization of visual matching by a bottlenosed dolphin (Tursiops truncatus): Evidence for invariance of cognitive performance with visual and auditory materials. Journal of Experimental Psychology: Animal Behavior Processes, 15, 124-136.

Herman, L. M., Richards, D. G., \& Wolz, J. P. (1984). Comprehension of sentences by bottlenosed dolphins. Cognition, 16, 129-219.

Herman, L. M., \& Thompson, R. K. R. (1982). Symbolic, identity, and probe delayed matching of sounds by the bottlenosed dolphin. Animal Learning \& Behavior, 10, 22-34.

HUNTER, G. (1988). Visual delayed matching of two-dimensional forms by a bottlenosed dolphin. Unpublished master's thesis, University of Hawaii, Honolulu.

JaCkson, W. J., \& Pegram, G. V. (1970). Comparison of intra- vs extradimensional transfer of matching by rhesus monkeys. Psychonomic Science, 19, 162-163.

JARRARD, L. E., \& MoISE, S. L. (1971). Short-term memory in the monkey. In L. E. Jarrard (Ed.), Cognitive processes of nonhuman primates (pp. 1-24). New York: Academic Press.

Mardon, K. A., \& Herman, L. M. (1987, June). Auditory delayed matching in a California sea lion (Zalophus californianus). Paper presented at the 1987 Animal Behavior Society Conference, Williamstown, MA.

Mello, N. K. (1971). Alcohol effects on delayed matching to sample performance by rhesus monkey. Physiology \& Behavior, 7, 77-101.

Nissen, H. W., Blum, J. S., \& Blum, R. A. (1948). Analysis of matching behavior in chimpanzee. Journal of Comparative \& Physiological Psychology, 41, 62-74.

Oden, D. L., Thompson, R. K. R., \& Premack, D. (1988). Spontaneous transfer of matching by infant chimpanzees (Pan troglodytes). Journal of Experimental Psychology: Animal Behavior Processes, 14, $140-145$.

Overall, J. E. (1980). Calculation of adjusted response frequencies using least squares regression methods. Applied Psychological Measurement, 4, 65-78.

Premack, D. (1978). On the abstractness of human concepts: Why it would be difficult to talk to a pigeon. In $\mathbf{S}$. H. Hulse, H. Fowler, \& W. K. Honig (Eds.), Cognitive processes in animal behavior (pp. 423-451). Hillsdale, NJ: Erlbaum.

Premack, D. (1983). The codes of man and beast. Behavioral \& Brain Sciences, 6, 125-137.

ROBERTS, W. A. (1972). Short-term memory in the pigeon: Effects of repetition and spacing. Joumal of Experimental Psychology, 94, 74-83.

RoITBLAT, H. L. (1980). Codes and coding processes in pigeon shortterm memory. Animal Leaming \& Behavior, 8, 341-351.

RoITBlat, H. L. (1987). Introduction to comparative cognition. New York: W. H. Freeman.

RoItblat, H. L., \& Scopatz, R. A. (1983). Sequential effects in pigeon delayed-matching-to-sample performance. Journal of Experimental Psychology: Animal Behavior Processes, 9, 202-221.
Schusterman, R. J. (1968). Experimental laboratory studies of pinniped behavior. In R. J. Harrison, R. C. Hubbard, R. S. Peterson, C. E. Rice, \& R. J. Schusterman (Eds.), The behavior and physiology of pinnipeds (pp. 87-171). New York: Appleton-Century-Crofts.

SChusterman, R. J. (1972). Visual acuity in pinnipeds. In H. E. Winn \& B. L. Olla (Eds.), Behavior of marine animals: Current perspectives in research: Vol. 2. Vertebrates (pp. 469-492). New York: Plenum.

Schusterman, R. J., \& Krieger, K. (1984). California sea lions are capable of semantic comprehension. Psychological Record, 34, 3-23.

Schusterman, R. J., \& Krieger, K. (1986). Artificial language comprehension and size transposition by a California sea lion (Zalophus californianus). Journal of Comparative Psychology, 100, 348-355.

Shyan, M. R., Wright, A. A., CoOK, R. G., \& Jitsumor, M. (1987). Acquisition of the auditory same/different task in a rhesus monkey. Bulletin of the Psychonomic Society, 25, 1-4.

SMITH, L. (1967). Delayed discrimination and delayed matching in pigeons. Journal of the Experimental Analysis of Behavior, 10, 529-533.

SoKAL, R. R., \& RoHLF, F. J. (1969). Biometry: The principles and practice of statistics in biological research. San Francisco: W. H. Freeman.

THOMPSON, R. K. R. (1981, October). Nonconceptual auditory matching by a rhesus monkey reflects biological constraints on cognitive processes? Paper presented at the Northeastern Meeting of the Animal Behavior Society, Kingston, Ontario, Canada.

WEINSTEIN, B. (1941). Matching-from-sample by rhesus monkeys and by children. Journal of Comparative Psychology, 31, 195-213.

WrIGHT, A. A. (1971). Psychometric and psychophysical hue discrimination functions for the pigeon. Vision Research, 12, 1447-1464.

Wright, A. A., Cook, R. G., Rivera, J. J., Sands, S. F., \& Delius, J. D. (1988). Concept learning by pigeons: Matching-to-sample with trial-unique video picture stimuli. Animal Learning \& Behavior, 16, 436-444.

Wright, A. A., Urculoli, P. J., \& Sands, S. F. (1986). Proactive interference in animal memory. In D. F. Kendrick, M. E. Rilling, \& M. R. Denny (Eds.), Theories of animal memory (pp. 101-125). Hillsdale, NJ: Erlbaum.

Wright, A. A., Shyan, M. R., \& Jitsumori, M. (1990). Auditory same/different concept learning by monkeys. Animal Leaming \& Behavior, 18, 287-294.

Zental, T. R., \& Hogan, D. E. (1978). Same/different concept learning in the pigeon: The effect of negative instances and prior adaptation to transfer stimuli. Journal of the Experimental Analysis of Behavior, 30, 177-186.

\section{NOTE}

1. D'Amato did not present sufficient data for one to judge whether first-trial transfer may have occurred as well.

(Manuscript received April 11, 1990; revision accepted for publication October 10, 1990.) 